\title{
Pensar el sorteo. Modos de selección, marcos deliberativos y principios democráticos*
}

\author{
Thinking Sortition Modes of selection, deliberative frameworks \\ and democratic principles
}

DIMITRI COURANT

\begin{abstract}
Resumen: ¿Cómo pensar el sorteo con eficacia? Mi propuesta es construir una teoría general del sorteo, abordándolo de forma comparativa, para averiguar cuáles son las constantes teóricas entre la gran diversidad empírica de sus usos particulares. En primer lugar, comparo el sorteo a los otros tres modos de selección: elección, nombramiento o cooptación y certificación. En segundo lugar, analizo los marcos deliberativos, es decir, "quién decide qué y cómo". Tercero, distingo cuatro principios democráticos del sorteo: igualdad, imparcialidad, representatividad y legitimidad. Mi primera hipótesis es que el sorteo amplía considerablemente la igualdad, imparcialidad y representatividad. La segunda de mis hipótesis radica en que el sorteo es el único método de selección con una forma específica de humildad-legitimidad, mientras que los tres restantes modos de selección producen una superioridad-legitimidad que declara superior al representante por encima del representado a través del proceso de selección.

Palabras clave: Deliberación, democracia, igualdad, imparcialidad, legitimidad, representación, selección, sorteo.
\end{abstract}

\begin{abstract}
How could we be thinking sortition efficiently? My proposal is to construct a general theory of sortition in a comparative approach. A broad study seems necessary in order to grasp the theoretical constants, despite the empirical diversity of sortition concrete uses. First, I shall compare sortition to the three other selection modes: election, nomination and certification. Second, I will analyse the deliberative frameworks, that is to say "who decides what how". Third, I will distinguish four democratic principles of sortition: equality, impartiality, representativeness and legitimacy. My first research hypothesis is that sortition is likely to offer a greater equality, impartiality and representativeness. My second hypothesis is that sortition is the only method of selection producing a specific form, a humilitylegitimacy when the three other selection modes are producing a superiority-legitimacy, where the principal is declared superior to the agent through the selection process.
\end{abstract}

Keywords: Deliberation, democracy, equality, impartiality, legitimacy, representation, selection, sortition.

Fecha de recepción: 01/06/2017. Fecha de aceptación: 25/09/2017.

* La traducción al español, a cargo de José Luis Bellón Aguilera, se ha hecho en el marco del proyecto de I+D: "La recepción de la Filosofía grecorromana en la Filosofía y las Ciencias Humanas en Francia y España desde 1980 hasta la actualidad" (FFI2014-53792-R).

** Dimitri Courant, University of Lausanne, IEPHI-LAGAPE, Géopolis, Bureau 4159, Lausana, 1015, Switzerland. Email: Dimitri.courant@unil.ch. Realiza el doctorado en Ciencia Política con una tesis titulada The New Spirit of Sortition. Representation and democratic principles in contemporary deliberative institutions. Entre sus publicaciones cabe destacar "Du klérotèrion à la cryptologie. L'acte de tirage au sort au XXIe siècle, pratiques et équipements" in Sintomer Yves et Rabatel Liliane (dir.), Tirage au sort et démocratie directe, La Découverte, 2017 y “Tirage au sort et délibération dans l’Armée française. Sociologie du Conseil Supérieur de la Fonction Militaire", Participations, n spécial, forthcoming 2018. 


\section{Introducción}

Tras siglos de ausencia el sorteo está regresando, a través de distintas investigaciones académicas, experimentos prácticos y reivindicaciones de activistas que vinculan participación y deliberación (Sintomer, 2011) ${ }^{1}$. Sin embargo, pensadores, experimentos y grupos de militantes divergen a la hora de explicar qué es el sorteo y cómo puede fortalecer la democracia. Mi propuesta persigue construir una teoría general del sorteo basada en modelos de tipo ideal, con el fin de llegar a una comprensión exhaustiva de esta variedad de hechos sociales.

Planteo una serie de preguntas recurrentes: ¿Quién puede ser sorteado? ¿Puede uno negarse a participar? ¿Exige la política algún tipo de competencias? Me sirvo de una perspectiva comparativa para exponer que la mayoría de las cuestiones que abordan el sorteo no se ocupan del sorteo como tal, sino del proceso de selección en general. Soy consciente de la existencia de una gran variedad de formas de sorteo y de elección, nombramiento y certificación, sin embargo, comenzaré por considerarlas como categorías relativamente coherentes. De lo que se trata es de mostrar que todos los modos de selección se enfrentan a los mismos problemas, pero responden basándose en principios diferentes.

¿Cómo podemos pensar el sorteo con eficacia? Mi hipótesis es que una forma productiva radica en abordar los modos de selección comparándolos, desde una perspectiva interdisciplinar. ¿Cuáles son los principios democráticos que forman el "nuevo espíritu del sorteo”? La historia nos ofrece, principalmente, tres significados sucesivos del sorteo: la selección de ciudadanos por sorteo para cargos públicos en Atenas, basada en el principio de igualdad; la asignación sorteada de miembros de jurados populares, con el fin de asegurar imparcialidad; por último, los sondeos de opinión, que aportan una representatividad de la población por medio de una muestra representativa. En un caso, el Consejo Superior de la Función Militar (CSFM), parece que los tres principios están ahí y se fusionan, produciendo y revelando un tipo específico de legitimidad que yo llamo "legitimidad de sorteo", "humildad" o "similitud", puesto que esta legitimidad es horizontal, neutral e impersonal. Difiere de las "legitimidades por superioridad" facilitadas por los otros tres modos de selección: elección, nombramiento (o cooptación) y certificación (Courant, 2014).

Mi investigación, analítica y normativa, se emplaza en el actual debate sobre el potencial y límites políticos del sorteo como "profundización democrática". Resulta necesario un estudio amplio centrado en comprender las constantes teóricas del sorteo, a pesar de la diversidad empírica de sus usos concretos ligados a instituciones y contextos en los que operan.

En primer lugar, compararé el sorteo a los otros tres modos de selección. En segundo lugar, distinguiré cuatro principios o potencialidades del sorteo: igualdad, imparcialidad, representatividad y legitimidad. Mi hipótesis es que el sorteo ofrece más igualdad, imparcialidad y representatividad. Sobre la legitimidad, mantengo que el sorteo es el único método de selección capaz de generar una forma específica de humildad-legitimidad, mientras que los tres restantes modos de selección producen una forma de superioridad-legitimidad, en la que el representante es declarado superior al representado a través del proceso de selección. Se trata de una teoría inductiva desarrollada gradualmente en el marco de investigaciones de campo (Courant, 2014).

1 La presente traducción sigue la bibliografía del autor, sin buscar ediciones españolas de los trabajos citados. [Nota del traductor.] 


\section{Modos de selección}

Una parte importante de los sistemas sociales se asienta en los procesos de selección. Estos se vuelven ineludibles en el momento en el que surge un bien, una tarea o una posición deseadas por demasiadas personas, o bien no deseadas pero necesarias para la colectividad. En la esfera política, dejando a un lado las pequeñas democracias directas, algunas misiones -en su mayor parte deliberativas- no pueden ser realizadas por todos los ciudadanos y necesitan ser cumplidas por representantes (Pitkin, 1972; Sintomer, 2013). Al comparar el sorteo con los otros tres modos de selección, cabe la posibilidad de que puedan combinarse, de que no sean incompatibles. Puesto que ya casi han desaparecido, dejo a un lado la filiación (herencia) y la adquisición (compra de cargos), y los modos de "autoselección", para concentrarme en procedimientos basados en la "heteroselección", especialmente en las desventajas de los modos de selección diferentes al sorteo.

\section{A) Elección}

La reciente victoria de la elección como el mecanismo legítimo para seleccionar gobernantes nos ha hecho olvidar que, con anterioridad a las revoluciones americana y francesa, la forma de pensar comúnmente aceptada era la siguiente: "El sufragio por azar (sorteo) es la naturaleza de la democracia. El sufragio por elección es la naturaleza de la aristocracia. Echar las suertes es una forma de elegir que no ofende a nadie, que permite a cada uno de los ciudadanos mantener una esperanza razonable de poder servir a su país" (Montesquieu, 1979, 134). Si bien los griegos inventaron la elección, el corazón de la democracia ateniense era el sorteo, mecanismo por medio del cual se asignaban la mayor parte de los cargos públicos, garantizando la igualdad de todos los ciudadanos -incluidos los "mejores" o aristoi-, excepto para ciertas tareas específicas, como los altos mandos militares (Hansen, 1991). Esparta, considerada como una oligarquía, se servía principalmente de la elección.

Manin (2012) explica que el sorteo era visto como democrático y la elección como oligárquica; más aún: tanto los Padres Fundadores de los Estados Unidos como los revolucionarios franceses odiaban de hecho la democracia hasta el punto de crear un gobierno representativo contra ella. Prefirieron la elección a la hora de seleccionar representantes, para crear una aristocracia elegida, socialmente distinta al pueblo. Posteriormente, la palabra democracia sirvió de herramienta propagandística con el fin de seducir a los electores, y nuestros regímenes cambiaron sus nombres, llamándose "democracias representativas" (Dupuis-Déri, 2013).

La elección es un procedimiento de selección de ascensión vertical de abajo arriba. Cada cuatro o cinco años, la minoría más numerosa del conjunto de los electores decide a cuál de los candidatos preseleccionados quiere entregarle el poder, quién le disgusta menos que gane de entre los dos o tres individuos preseleccionados. La elección presenta tres inconvenientes: 1) No ofrece una buena representatividad de los representados. 2) Genera un tipo de legitimidad personal e individual. 3) No garantiza la competencia en la deliberación, ya que importan más el carisma y la comunicación que los resultados y las promesas lanzadas en manifiestos, que no son vinculantes puesto que el mandato imperativo está prohibido. 


\section{B) Nombramiento (o cooptación)}

El nombramiento es una forma de selección realizada por un agente, mientras que en la cooptación participan varios, pero no demasiados. Parece un modo de selección, en principio, sospechoso y obsoleto. Sin embargo, este "ordeno y mando" irrestricto y no sujeto a discusión o debate, es una práctica muy generalizada en nuestros gobiernos representativos. De hecho, en Francia, el Primer Ministro es nombrado por el Presidente, el cual es elegido por medio de sufragio universal directo. El Primer Ministro nombra a su vez a los ministros de su gobierno y el Presidente tiene que dar su aprobación, lo que es una forma de cooptación. En ninguna parte se elige a los miembros de un gobierno, aparte del referido Primer Ministro o, en algunos países, el Canciller.

Al nombramiento/cooptación: 1) Se le puede acusar de parcialidad, sesgo y arbitrariedad; 2) No ofrece una buena representatividad de los representados: una persona nombra a sus conocidos de círculos relativamente homogéneos; 3) Su forma de legitimidad es de descenso vertical de arriba abajo, creando hostilidad en la base; 4) Genera una forma de legitimidad muy personalista e individual.

\section{C) Certificación}

La certificación es uno de los procesos de selección más utilizados (por universidades, funcionarios, etc.), pero casi nunca se emplea para elegir representantes. Parecería, por tanto, que la representación no es una acción que requiera competencias certificadas. La representación puede ser aceptada de dos maneras: autorización o identificación. Los representados, o bien autorizan al representante a hablar en su nombre, o bien se identifican con un representante parecido a ellos. La autorización es, con frecuencia, el resultado de una elección, pero no necesariamente (Saward, 2010). La identificación a veces resulta de un sorteo que produce una muestra representativa parecida al grupo representado.

La certificación: 1) Es incapaz de proveer ni autorización ni identificación; 2) Presenta el principio de distinción: los representantes no se parecen a los representados (como en la elección y nombramiento/cooptación). 3) Genera una forma de legitimidad individual basada, al mismo tiempo, en la superioridad. A pesar de que la certificación parece garantizar cierta igualdad de oportunidades entre candidatos e imparcialidad en el examen, lo que generaría un tipo de legitimidad horizontal, las tres cualidades señaladas son relativas. En realidad, las modalidades de examen son decididas por superiores que crean una categorización parcial, exigiendo cualidades que solo poseen algunos candidatos. Si bien la legitimidad no procede tan directamente de arriba como en el nombramiento, puede considerarse como de semi-descenso vertical, al provenir de una como quasi-cúpula. Si bien la cúpula no elige directamente a su candidato favorito, crea una prueba en la que es más probable que esos candidatos puedan ganar.

\section{D) Sorteo - competencia y eficacia}

La eficacia no es un "principio democrático del sorteo", puesto que no constituye per se un valor democrático. Sin embargo, defenderé que el sorteo es potencialmente más eficaz 
que otros modos de selección. La principal crítica que se le hace al sorteo proclama que produce la selección de incompetentes, a pesar de que "el sorteo nunca ha favorecido a la gente incompetente por encima de los competentes" (Rancière, 2005: 49). Incluso considerando que el sorteo no es un filtro de competencia, sería erróneo creer que otros modos de selección garantizan en sí mismos el escogimiento mecánico de actores competentes. Sólo la certificación puede pretender hacerlo, a condición de que pruebas y exámenes estén "correctamente establecidos", algo sujeto a controversia. Pero los agentes a cargo de seleccionar representantes, situados en la cúpula si es por nombramiento o en la base si por elección, podrían equivocarse de medio a medio en cuanto a las competencias reales de los candidatos. Cabe incluso señalar que esto sucede a menudo, si se considera la cantidad de escándalos provocados por líderes incompetentes y corruptos. Aquellos que se presentan voluntariamente o se auto-seleccionan para ser candidatos se ven a sí mismos como competentes, aunque podrían no serlo y su confianza en sí mismos sería en realidad arrogancia. En cambio, aquellas personas que se ven a sí mismas como indignas de presentarse, podrían poseer la principal cualidad del ciudadano según Aristóteles (2009): la prudencia.

Por otro lado, en la gran mayoría de los experimentos de democracia deliberativa, los ciudadanos seleccionados pasan por una formación intensiva (Gastil \& Levine, 2005). Desde la nanotecnología a la constitución, los casos muestran que los ciudadanos aprenden deprisa y que en poco tiempo adquieren más competencias en cuestiones complejas que los cargos electos (Boy \& Bourg, 2005).

Finalmente, debido a la "diversidad cognitiva" que proporciona, la selección por sorteo puede ser un "modo epistémicamente superior de selección de representantes", puesto que "las decisiones tomadas por muchos probablemente serán más acertadas que las decisiones tomadas por unos pocos". De hecho, el sorteo ofrece una asamblea poblada de una mayor diversidad de experiencias y perfiles sociales, creando una inteligencia colectiva más fuerte (Landemore, 2013).

\section{Marcos de selección y deliberación}

Necesitamos analizar a qué desafíos compartidos se enfrentan los diferentes modos de selección: "quién decide qué y cómo". Se responden así las preguntas planteadas al sorteo, avanzando, además, hacia la cuestión de qué es lo realmente específico en la selección sorteada. Un argumento usado con frecuencia contra el sorteo sostiene que es irracional ponerse en manos de la suerte y que los individuos no quieren perder la posibilidad de elegir. Sin embargo, incluso si se usa el sorteo, hay muchas decisiones que tomar sobre el porqué y el cómo organizar las suertes y la deliberación (Dowlen, 2008; Stone, 2011).

\section{A) Mandato e instituciones}

\section{Misión}

"Representación" y "deliberación" cubren un amplio abanico de significados y realidades. "Representar" significa tener la capacidad de "hablar y/o actuar por" un grupo (Burdeau, 1966; Sintomer, 2013). Antes de elegir el modo de selección se debería plantear 
la siguiente pregunta: “¿Qué tarea hay que realizar? ¿Qué tipo de portavoz queremos?”. Si se quieren representantes que se parezcan a los representados, una "representación descriptiva" (Pitkin, 1972), elegiremos el sorteo, acercándonos al ideal democrático del "gobierno del pueblo". Si preferimos élites socialmente diferenciadas, una perspectiva aristocrática, optaremos por la elección. Si se cree que son los líderes los que deberían elegir a los representantes, adoptaremos -dentro de la perspectiva oligárquica- el nombramiento. Si se buscan representantes cualificados, elegiremos a través de la certificación, inclinándonos por la tecnocracia.

La mayoría de los teóricos, activistas, así como los resultados de experimentaciones, indican que las tareas de una asamblea elegida por sorteo deberían ser deliberativas, no ejecutivas. Las siete tareas principales son las siguientes: 1) Consulta a la población, como en una muestra deliberativa (Fishkin \& Luskin, 2005); 2) Información de funcionarios y ciudadanos por medio de comunicados (Gastil \& Richards, 2013); 3) Control y evaluación del gobierno, funcionariado y políticas (Sintomer, 2011); 4) Decisión, como en ciertos presupuestos participativos; 5) Legislación, como una cámara adicional en el Parlamento (Sutherland, 2008); 6) Constitución, tanto para reformar como para redactar un nuevo texto (Reuchamps \& Suiter, 2016); 7) Problemas a largo plazo, tales como el cambio climático o riesgos tecno-científicos (Bourg, 2011). Estos cometidos no son mutuamente excluyentes.

\section{Estructura}

¿Qué arquitectura institucional integra a los representantes? ¿La usaremos para grupos pequeños o para una gran asamblea? Los principales experimentos del sorteo han sido o bien un jurado de 12 a 30 ciudadanos, o una asamblea más amplia, 160 en la Columbia Británica y 1.200 en Islandia (Sintomer, 2011, 161-189). Esos congresos y asambleas eran de carácter consultivo y nunca tomaban la decisión final, dejando esta al parlamento electo o llevándola directamente a referéndum.

La reivindicación más importante de los activistas del sorteo está vinculada a la reforma electoral y a la Constitución. Señalan -certeramente- que no se debería permitir a las personas electas cambiar las reglas de su propio control, para evitar conflictos de intereses. Una Convención Constitucional, elegida por sorteo entre ciudadanos corrientes, concedería a los propios ciudadanos más peso y formas de intervención política tales como referéndums y otros mecanismos de participación, fortaleciendo el control del pueblo sobre los individuos electos.

Académicos y activistas abogan por una asamblea de miembros sorteados como tercera cámara del Parlamento o como sustitución de una de ellas. Esto tiene la ventaja de mantener las elecciones y a los partidos en una cámara, logrando con la otra una representación real de la diversidad popular (Sutherland, 2008). Como ya apuntó Dahl (1989), el sorteo es propuesto casi siempre como un complemento añadido a la elección y no como un sustituto absoluto.

Las reglas que estructuran la deliberación constituyen, por otro lado, un tema fundamental. El análisis de las experimentaciones concretas aporta información sobre cómo facilitar la discusión y el voto, al tiempo que nos recuerda los problemas a los que se enfrentan (Gastil \& Levine, 2005). 


\section{Duración}

La duración y límites del mandato son importantes. Como dijo Aristóteles: "Se considera democrático distribuir los cargos por sorteo, y oligárquico para ellos el ser elegidos (asignados mediante voto)", a lo cual, sin embargo, añadía: "Una característica de la libertad es gobernar y ser gobernado por turnos". El sorteo puede ser usado para fines no-democráticos; por ejemplo, designar al azar un monarca absoluto, como en las novelas de ciencia-ficción (Dick, 1955). Si el sorteo permite formar asambleas con una sección razonablemente representativa de la población y una vecindad de experiencias vitales entre los representantes y los representados, el hueco entre ellos puede abrirse ex post. Los partidos comunistas insistieron siempre en que sus líderes tenían orígenes proletarios, pero una vez que se situaron a la cabeza del partido, nunca volvieron a las fábricas. Michels (1971), en su teoría de la "ley de hierro de la oligarquía”, analizó en 1911 esta tendencia a la falla entre representantes y representados.

La historia muestra que en todo sistema político basado en el sorteo, había siempre un mandato corto y un principio de rotación ${ }^{2}$. Ya se trate de la antigua Grecia, la Italia medieval, los jurados populares o los recientes mini-públicos, los mandatos de los representantes seleccionados por sorteo eran de corta duración (Sintomer, 2011). El sorteo y la brevedad del mandato permite una rápida rotación de representantes; por contraste, la elección favorece la reelección, la reproducción de certificaciones entre los aristoi es fácil y el nombramiento se convierte en patrimonio de pequeños círculos de iniciados. La temporalidad es crucial a la hora de esquivar la "oligarquización”. El ejemplo histórico más destacable, por combinar sorteo, corta duración de mandatos y rotación para evitar la profesionalización de la política, es la democracia ateniense, en la que se restringía a un año, una vez en la vida, la membrecía en el Consejo de los Quinientos (Hansen, 1991).

\section{Control y rendición de cuentas}

El control ejercido sobre los miembros electos constituye otro punto vital para una representación democrática. De nuevo, Atenas presta un interesante ejemplo, pues allí se vigilaba de cerca a las personas con un cargo público sorteado o electo, pudiendo ser, en cualquier momento, despedidas y condenadas por el pueblo. La rendición de cuentas no era una broma, ya que las sanciones oscilaban entre las pequeñas multas, el exilio o la ejecución.

Para muchos académicos, el momento de la rendición de cuentas en los gobiernos representativos tiene lugar en la reelección (Manin, 2012), lo que resulta tremendamente problemático. En primer lugar, puesto que los representantes no deberían quedarse en el poder demasiado tiempo, volviendo a presentarse a una reelección. En segundo lugar, puesto que algunos mandatos están limitados, como el de Presidente de los Estados Unidos, ello significaría que no habría que rendir cuentas tras el segundo término. Tercero, porque lo peor que le podría suceder a un político terriblemente decepcionante que hubiera mentido durante su campaña haciendo luego lo contrario de lo que prometió, sería simplemente que

2 Al contrario de los usos religiosos del sorteo (Courant, 2017). 
no ganara un segundo mandato. En este sentido, las instituciones básicas de un sistema democrático deberían ser el referéndum revocatorio popular, el control ciudadano durante el mandato, una escrupulosa rendición de cuentas y sanciones.

\section{B) Censo del sorteo}

\section{Criterios}

Sea cual sea el modo de selección o la esfera, existe siempre una delimitación del "cuerpo político relevante" que determina qué criterios te permiten formar parte del "grupo (o censo) a sortear", del proceso de selección. En nuestros sistemas actuales, participar en una elección -como electores o como candidatos- está restringido únicamente a los ciudadanos. Esto significa cumplir una serie de criterios: 1) edad, 2) nacionalidad, 3) integridad (no haber sido condenado por delitos graves), 4) independencia (no estar bajo tutela). Hasta hace poco, el sexo, la "raza" y aún antes la riqueza (capacidad de pagar una cuota impositiva) fueron también criterios de acceso a la ciudadanía.

En la mayor parte de los experimentos y teorías del sorteo, el grupo a sortear está formado por todos los ciudadanos de una circunscripción electoral, si bien algunas veces puede ser más inclusivo. En determinados casos se ha usado el listín telefónico, alcanzando por tanto a individuos sin registrar en las listas de voto. El G1000 belga reservó incluso el $10 \%$ de los asientos a vagabundos e inmigrantes, con el fin de lograr la mayor diversidad e inclusión posibles (Reuchamps \& Suiter, 2016). El grupo a sortear está relacionado con el nivel en que se implementará la decisión en cuestión, desde un vecindario local a un Estado, un continente (Buchstein \& Hein, 2010) o todo el mundo, como en las reuniones de 10.000 ciudadanos en los sondeos deliberativos World Wide Views del evento de París COP21.

\section{Selección mixta}

Cabe la posibilidad de no considerar los modos de selección como incompatibles, sino como complementarios. Es posible combinar varios para una misma designación. En la actualidad, los candidatos para la elección han sido ya seleccionados por sus partidos, mediante cooptación o primarias. En el ejército, para convertirse en miembro del CSFM se requiere a los soldados que obtengan una certificación correspondiente a sus rangos, luego ser sorteados y, desde 2005, ser también elegidos por las demás personas designadas por sorteo (Courant, 2014). Se podría plantear que hace falta algún tipo de examen para formar parte del grupo a sortear, pero este argumento aristocrático va en contra de la igualdad democrática encarnada en el principio "una persona, un voto".

Con el objetivo de poner de manifiesto la flexibilidad de la selección mixta y para estimular la imaginación democrática, voy a proponer lo siguiente: los partidos políticos presentan sus manifiestos y largas listas de candidatos, los ciudadanos votan el manifiesto, a continuación se realiza un sorteo en las listas del partido ganador para seleccionar la proporción de representantes dependiendo de cuántos escaños ha ganado ese partido; y un examen de certificación para conseguir el derecho a ser nombrado Ministro. Se pueden imaginar un número enorme de combinaciones. 


\section{C) Dínamis}

Para eliminar la ambigüedad presente en la cuestión de la "participación voluntaria", tuve que crear el concepto de inflexión o dínamis, el cual expresa el grado de influencia que un designado o potencial designado puede ejercer sobre su propia designación. Distingo tres niveles: una dínamis (o grado de inflexión) fuerte en el voluntariado, una dínamis débil en el consentimiento y una inexistente en el deber (ibid.). Va más allá de la mera distinción entre autoselección y heteroselección.

\section{Voluntariado}

En este primer nivel, el sorteo es realizado entre voluntarios y el censo está compuesto sólo de candidatos. Este método tiene la ventaja de que los participantes son personas motivadas, con la desventaja de que el poder es dejado en manos de aquellos que lo querían. Esto va en contra de filósofos como Platón (2005) o Rancière (2005), para los que "el peor de los males es dejar que las personas que desean el poder lo consigan". Sin embargo, el sorteo entre personas voluntarias era el modo de funcionamiento en Atenas, Venecia, Florencia y Aragón (Sintomer, 2011). Esta etapa de voluntariado introduce una "reivindicación representativa" (Saward, 2010).

\section{Consentimiento}

Es el segundo nivel de la dínamis: el sorteo es realizado entre el conjunto del "cuerpo político relevante", sin convocatoria alguna de voluntarios. Se sortean todos los miembros de los grupos, si bien aquellas personas que sean seleccionadas tienen el derecho a rechazar el cargo público a posteriori. La gran mayoría de experimentaciones sobre la democracia deliberativa funcionan con este modelo.

El consentimiento conduce a la ruptura de la igualdad y de la representatividad, aunque en menor medida que el voluntariado. Al dejar a cada persona que decida, obtenemos una muestra que no se parece al grupo más amplio que queremos representar. Algunas categorías brillan por su ausencia, puesto que estas personas no se consideran a sí mismas tan iguales como otras, no lo suficientemente buenas, indignas de participar. Con frecuencia, los varones adultos blancos, ricos y bien educados aceptan participar, mientras que las mujeres, los jóvenes y los pobres se niegan. La misma tendencia se da en las elecciones: las clases sociales no privilegiadas no votan, revelando el "censo oculto" (Gaxie, 1993).

Esto es problemático a dos niveles. Primero, a nivel ético: la ausencia de los "dominados" va en contra de la justicia y la inclusión. Segundo, a nivel pragmático: se crea una falta de legitimidad y de eficacia, ya que algunos de aquellos ciudadanos de "segunda categoría" pueden acabar por perderle el respeto a leyes sobre las que nunca son consultados cuando se promulgan.

\section{Deber}

El último y más débil nivel de la dínamis es el deber: se realiza un sorteo entre el conjunto del "cuerpo político relevante" sin pedir voluntarios ni permitir el rechazo del cargo 
ex post. En nuestras sociedades liberales, resulta extraño ver la participación como un deber. Sin embargo, es práctica común en Francia, Estados Unidos, Gran Bretaña, etc., en los jurados populares. El Estado selecciona por sorteo a los ciudadanos que formarán parte obligatoriamente de los jurados (Leib, 2004). El deber es uno de los fundamentos de la vida en sociedad, ya sea la obligación de dar una educación a los niños o de pagar los impuestos. Más aún, este sistema de sorteo, unido a un deber-dínamis, es perfecto para la justicia, que tiene que garantizar la imparcialidad de los que deciden en el juicio. Elegir un jurado de entre candidatos voluntarios levantaría, sin duda, muchas sospechas, puesto que cualquiera acabaría preguntándose si en realidad hay intereses en juego que motivan su participación.

El deber protege asimismo contra el fenómeno del "gorrón". Participar en una asamblea o jurado deliberativo supone un gasto enorme en tiempo y energía cuyos costes individuales reportan beneficios a la colectividad. Un actor racional egoísta podría llegar a la conclusión de que participar no le vale para nada y que es mejor dejar a otros este tipo de tareas; sin embargo, esta persona se beneficiaría del trabajo de aquellos (Olson, 2011). Esta estrategia del gorrón se vuelve imposible con un sistema mix to de sorteo y deber. De hecho, constituye una de las razones por las que el sorteo fue aplicado en la Inglaterra del siglo XVIII, debido a que los notables solían pagar para evitar servir en los tribunales.

Finalmente, el deber tiene la ventaja de combatir simultáneamente tanto la "autoexclusión" como la "heteroexclusión". La autoexclusión es el "censo oculto": algunos individuos se impiden a sí mismos la participación porque se ven a priori como indignos. Obligarles les impulsaría a participar y a darse cuenta de que son absolutamente capaces, lo que genera empoderamiento.

La heteroexclusión sucede cuando una serie de elementos externos bloquean la participación de personas o grupos. Como en Estados Unidos, donde las largas distancias hasta las mesas electorales, las largas colas y la falta de papeletas desalentaba a muchos ciudadanos a la hora de votar, sobre todo a las personas de color y a los pobres. Igual que es imperativo entrar en una cabina para ejercer el voto libre de amenazas, el voto obligatorio reduce las posibles presiones. La obligación hace a las personas libres. El hecho de que el ciudadano elegido por sorteo no pueda rechazar su misión elimina el riesgo de presiones externas, de autoexclusión y garantiza la igualdad, imparcialidad y representatividad de la muestra.

El sorteo por sí mismo no es suficiente. Para ser democrático, o "demoskrático"3, debe combinarse con: 1) El hecho de que el sorteo da una tarea y no el poder, 2) Colegialidad de la asamblea de deliberación colectiva, 3) Mandatos cortos y rotación rápida, 4) Rendición de cuentas y control, 5) Restricciones mínimas para la integración en el proceso de selección.

\section{Principios democráticos}

Los principios democráticos del sorteo son, en mi opinión, cuatro: igualdad, imparcialidad, representatividad y legitimidad, cada uno de ellos subdivididos en tres elementos (Courant, 2014). Gracias a esos principios, el sorteo puede producir un tipo mejor de representación, deliberación y participación. Sin embargo, el sorteo no tiene una naturaleza

3 "Demoskrático": neologismo a partir de demos ("pueblo") y kratos ("poder"), con el sentido fuerte de "democracia real". [Nota del traductor.] 
única: sus principios formales pueden ser enriquecidos o perjudicados dependiendo de la arquitectura institucional en la que se integre. Esos principios son potencialidades, no están todos siempre presentes cuando el sorteo entra en uso, ni siquiera con la misma intensidad. Con todo, comparadas esas potencialidades con las producidas por otros modos de selección ceteris paribus, en un marco de deliberación similar, los cuatro principios democráticos se fortalecen con el uso del sorteo.

\section{A) Igualdad}

\section{Igualdad estadística, para cada miembro del grupo}

"La unión entre igualdad política y deliberación se remonta a la antigua Atenas, donde un microcosmos deliberativo de varios cientos de ciudadanos, seleccionados por sorteo, tomaba decisiones cruciales", escriben Fishkin \& Luskin (2005), al presentar la inspiración del sondeo deliberativo. Sin voluntariado ni cuotas, el sorteo proporciona una igualdad estadística excelente entre los individuos. Cada ciudadano tiene las mismas oportunidades de salir elegido por la suerte, y sin intervenir ningún tipo de discriminación, positiva o negativa.

Para Castoriadis (1996), este principio equivale al sufragio universal y al gobierno de la mayoría: todos los ciudadanos son considerados iguales en competencia política, todas las voces son consideradas iguales. Atacar el sorteo aduciendo que no deberíamos encomendar tareas de representación y deliberación a cualquiera, equivale a decir que no deberíamos conceder el derecho de voto a todos. "Democracia significa (...) poder de los que no tienen derecho a gobernar ni a ser gobernados", plantea Rancière $(2005,54 ; 56)$. "El escándalo de la democracia y del sorteo -esencia de la democracia- consiste en revelar que el poder del pueblo es (...) el poder de cualquiera".

Es probable que los ciudadanos sean más competentes votando ideas y problemas que a candidatos. Mucho más si los ciudadanos tienen la oportunidad de pasar por un procedimiento "clásico" de democracia deliberativa, con formación, lecturas, debates en grupos, etc. En comparación con el votante estándar, enfrentado a infoespectáculos ${ }^{4}$, la competencia política de un participante en una asamblea sorteada es seguramente mejor.

\section{Igualdad deliberativa entre representantes}

El segundo tipo de igualdad producida por el sorteo es la igualdad deliberativa de los representantes. Una vez elegidos, todos los representantes son iguales entre sí. El sorteo elimina la afirmación de superioridad: "He ganado la elección con la mayoría más numerosa", "las autoridades me nombraron a mí en primer lugar", o "he pasado el examen con la mejor nota". Todos los representantes elegidos por sorteo tienen exactamente la misma posición, y no pertenecen ni a una mayoría ni a una oposición. Todas las voces deben ser escuchadas con idéntica atención, llevando a una deliberación más inclusiva, diversa y, por encima de todo, mejor (Landemore, 2013).

4 Lit. "Infotainment", del inglés "information" más "entertainment". [Nota del traductor.] 


\section{Igualdad inclusiva, para los representados}

El sorteo puede crear una igualdad inclusiva entre representantes y representados. De hecho, estos pueden perfectamente decirles a aquellos: "Somos iguales. La única diferencia entre nosotros es la suerte". "El efecto de inclusión no resulta sólo de que las personas sorteadas tengan las mismas posibilidades de salir elegidas, sino también del hecho de que cada uno de ellos sabe que podría ser él o ella" (Delannoi, 2010, 19). Sin duda esto aumenta la participación. El sorteo no elimina la participación, por el contrario, conduce a debates y votaciones de leyes por medio de referendos. Impide que se les espete a los ciudadanos: "Ya habéis participado en política, durante las elecciones. Habéis entregado vuestro poder a aquellos que elegisteis, así que permaneced en silencio mientras ellos hacen su trabajo". Las experimentaciones deliberativas basadas en el sorteo están vinculadas con frecuencia a procedimientos de democracia directa y participativa: debates públicos, participación por internet o referendos. Estas herramientas políticas empoderan a los ciudadanos más que la comunicación espectacular de las campañas electorales. Y puesto que el sorteo proporciona una muestra representativa, posiblemente ajustada con las cuotas, las minorías excluidas tendrían un porcentaje justo de puestos, creando un sentimiento de inclusión.

\section{B) Imparcialidad}

\section{Neutralidad: garantía contra la manipulación y la discriminación}

La imparcialidad -“la justicia ciega”- parece la cualidad más obvia del sorteo. Probablemente sea esta la razón por la que el uso más antiguo del sorteo que todavía pervive sea el jurado popular. La imparcialidad es también el principio básico que justifica el sorteo en las conferencias de ciudadanos o conferencias de consenso, particularmente en cuestiones tecno-científicas. Hay una tensión entre intereses tales como la salud pública o los beneficios económicos y es necesario consultar la opinión imparcial de ciudadanos "ordinarios" que han pasado una preparación contradictoria. La experiencia de muchas conferencias de ciudadanos proporciona ejemplos de formación imparcial y procedimientos deliberativos sobre cuestiones complejas (Boy \& Bourg, 2005). Consultar únicamente a expertos, activistas o industriales sembraría la duda sobre la credibilidad del proceso, por sospechas de conflicto de intereses o falta de objetividad. ¿Cómo seleccionar personas para este congreso sino a través del sorteo? El simple hecho de que un individuo sea un candidato para un jurado es de por sí sospechoso. El sorteo favorece que la gente que participa en una deliberación no tenga intereses ocultos en la cuestión, siendo todos parte de la sociedad y buscando el bien común. Asimismo, previene el amiguismo, las redes de contactos y las negociaciones de pasillo o antecámara entre grupos pequeños pero poderosos, ya que no se sigue ninguna "línea del partido" ni a patrocinadores o financiadores en las negociaciones. Pero incluso aquí, a pesar de todo, se establecerán procedimientos de control con el objetivo de evitar ex post la influencia ilegal de los lobbies. 
El sorteo vuelve inútiles tanto la manipulación de los medios de masas como el marketing político en la competición por escaños en la Asamblea. Sin embargo, los partidos y los medios todavía juegan un papel importante en cómo se debate una cuestión en la esfera pública y durante la campaña de referéndum después de la asamblea de deliberación. El sorteo limita el soborno y las medidas demagógicas para cazar electores.

Sea física o virtualmente -mediante un código fuente que cualquiera puede consultarel sorteo es un proceso fácilmente transparente (Courant, 2017); se acabaron las papeletas "olvidadas" o los cálculos erróneos de votos. Con todo, hablar de "transparencia" en el sorteo es ambiguo debido a la "zona ciega" o blind break (Dowlen, 2008). "Sé que algo está sucediendo, se está sorteando, pero no sé lo que está pasando porque no puedo predecir o influenciar el resultado". La suerte es arracional -no irracional- elimina las discriminaciones, "no sólo el cálculo 'racional', sino también cualquier tipo de cálculos: sin emociones ni prejuicios... sin buenas o malas razones" (Delannoi, 2010, 20), todo lo cual produce un "efecto sanitario" (Stone, 2011).

\section{Unidad: prevenir la competición}

La competición y la estrategia partidista no tienen sentido en el sorteo. Para esta cualidad, "la paz creadora de la virtud de exterioridad", fueron usados los sorteos en las repúblicas italianas, como forma de resolución de conflictos, para evitar "la violencia generada por la competición electoral abierta" (Manin, 2012, 74-93). Los sorteos son incontestables y evitan las campañas electorales, la demagogia y el faccionalismo.

Bourdieu ofrece una definición implacable de los partidos políticos, aplicable también a los sindicatos: "Los agentes de la lucha simbólica para la conservación o transformación de la palabra social son los partidos; organizaciones de combate especialmente diseñadas para librar esta forma sublimada de guerra civil" (Bourdieu, 1981). Algunos críticos de las polarizaciones partidistas como Weil (1957) o Lazure (2006) denuncian con frecuencia este riesgo. En el caso del ejército, el miedo a la división y el faccionalismo se incrementa considerablemente. De hecho, el imperativo permanente de asegurar la defensa de la nación no puede permitir la competición electoral. Si se deben escuchar todas las voces, especialmente aquellas que provienen de la base, en el proceso de diálogo, la selección de representantes no puede poner en peligro la unidad del ejército. Esta es la razón por la que el Parlamento francés aprobó el sorteo para el CSFM en 1968 (Courant, 2014).

Sin embargo, los debates contrapuestos entre representantes y ciudadanos son importantes. "Unidad" no significa aquí ausencia de divisiones, sino ausencia de escisiones preestablecidas provocadas por la afiliación a un partido antes de cualquier debate. A partir de cuestiones concretas, leyes que votar, deberían producirse nuevas divisiones y debates, algo que permanece con el sorteo. Lo que se destierra son las viejas -y a menudo artificialesfracturas partidistas, que necesitan distinguirse unas de otras a través de la comunicación. Desaparecida la disciplina de la "línea del partido" mejora la deliberación, puesto que cada representante puede deliberar de verdad, escuchar a otros, cambiar de opinión y votar con la cabeza, y no siguiendo órdenes de arriba.

Incluso sin partidos, la elección crea una forma de legitimidad personal, vertical, desigual y parcial, ya que un candidato puede elegir como interlocutor a una parte del 
electorado y olvidarse, o dejar a un lado, a otra. Por contraste, el sorteo -horizontal- desarrolla imparcialidad en el representante. La ausencia de prejuicios a priori permite a los representantes seleccionados por sorteo, liberados así de la ideología de partido, el buscar el bien común en vez de dedicarse a estrechos intereses de facciones.

\section{Lo imprevisible: crear un cambio político real}

La ausencia de parcialidad crea un representante que no trapichea puestos con el interés general, algo que suele reprochársele a los partidos. Además, la profesionalización de la política tiende a crear tipos políticos cortados por el mismo patrón. Siguen los códigos y reglas de sus cerrados círculos y se vuelven cada vez más previsibles:

El sentido del juego político que permite a los políticos predecir las posiciones de otros políticos es también lo que los convierte en previsibles. Previsibles y responsables, lo que significa competentes, serios, fiables, dispuestos a jugar el juego con constancia, sin sorpresas o traiciones al rol impuesto por la estructura del juego. Nada es más absolutamente requerido por el juego político que esta adhesión fundamental al juego mismo. (Bourdieu, 1981: 6-7)

Para Kontos (2001), la imprevisibilidad constituye la característica básica del sorteo. La política económica de la antigua Atenas era previsible porque los magistrados no lo eran, al contrario que las elecciones contemporáneas, que vuelven predecibles las estrategias de los políticos y volátil e incierta la esfera de la economía. Lo imprevisible es una forma de "velo de ignorancia", puesto que el sorteo evita que los participantes sepan cuáles son las posiciones de otros con relación a cualquier cuestión y también sus habilidades para deliberar o cambiar de opinión. Por tanto, cuando la deliberación empieza, nadie sabe qué camino tomará. Algunos mini-públicos, de hecho, acabaron produciendo cambios políticos sorprendentes e importantes, en materia de energías renovables en Texas (Fishkin, 2003) o sobre el matrimonio homosexual en Irlanda.

\section{C) Representatividad}

La hegemonía del sistema de elecciones produce la ilusión de que la representación por autorización es la única vía legítima. Por contraste, el sorteo se basa en la representación por identificación. El primer sistema produce una representación-distinción, mientras que el segundo una representación-descripción.

\section{Semejanza con el representado en la muestra representativa}

La voluntad de que haya similitud entre representantes y representados ha existido durante toda la historia del gobierno representativo. Los gobiernos representativos se fundaron sobre la elección y su "principio de distinción". La representación iba de la mano de la autorización y no de la descripción o identificación. Pitkin (1972) presenta dos niveles de representación y dos opciones para cada uno de ellos: 1) Defender a. a) Microcosmos: 
la asamblea es un conjunto similar al amplio grupo que representa. b) Agente-principal: los representantes son elegidos por sus habilidades, independientemente de sus características sociales y físicas, de modo que un hombre blanco y rico puede representar a un grupo de mujeres pobres de color. 2) Actuar en nombre de. a) Delegado: el representante es sometido a mandato imperativo y debe consultar al grupo antes de tomar cualquier decisión en su nombre. b) Administrador: el representante tiene la confianza del grupo y las manos libres para tomar cualquier decisión sin necesidad de consultar antes al grupo.

El sorteo activa el viejo ideal de la representación espejo, simplemente porque el conjunto de representantes provee una sección razonablemente diversificada de la población, una visión proporcional del grupo sorteado, en términos de clases sociales, edad, género, etc. (Sintomer, 2011, 147-190). Además, concede puestos a ciudadanos ordinarios. De esta forma, un representante sorteado tendría un origen social semejante al de la población que representa.

\section{Diversidad y representación colectiva}

"El Parlamento debería ser como un mapa para el territorio, un retrato en miniatura del Pueblo", dijeron Condorcet y los anti-federalistas americanos. Pero en la actualidad, aparte de la diversidad meramente geográfica, las elecciones producen asambleas que no se parecen a la población. En Francia en 2013, la mitad de la población activa estaba formada por empleados, porcentaje que solo llegaba al $3 \%$ entre los diputados. Esta falta de diversidad va contra los antiguos principios del quod omnes tangit, que no significa otra cosa sino que todos deberían discutir cuestiones que conciernen a todos. Esta idea la expresa Dewey (1929, 207) con otras palabras: "El que lleva los zapatos sabe si le aprietan y dónde". Igual que en el argumento epistémico, la diversidad de una asamblea representativa es también una cuestión de eficacia (Landemore, 2013).

Incluso si el tamaño de la asamblea es demasiado pequeño como para tener una muestra representativa decente, igual que en un jurado, el sorteo está diseñado para lograr la mayor diversidad posible. Algunas experimentaciones se esfuerzan por atraer e incluir personas de colectivos marginados, como los indígenas en Canadá5. Como mostró Phillips (1995): "La composición de género o étnica (...) de las asambleas se convierte en un asunto legítimo de preocupación democrática".

La llamada "diversidad de partido" es en realidad muy débil, especialmente en los sistemas bipartidistas. Un gran número de preocupaciones de la población no están representadas y la afiliación y apoyo a partidos decrece. La representatividad que implica el sorteo es necesariamente colectiva; la asamblea entera representa a la población como un todo coherente, no a cada miembro individualmente. Por su parte, una persona electa puede verse a sí misma como un "representante personal" con su propia agenda, puesto que cuenta con la autorización de haber sido elegido por la minoría más fuerte.

5 La determinación de sub-poblaciones relevantes debería abrirse al debate político. 


\section{Proximidad a la base}

Si el sorteo logra una semejanza significativa con la población, puede suceder que la distancia entre representantes y representados crezca ex post. Sin embargo, en todos los casos políticos, el sorteo siempre se concierta con mandatos cortos y rotación. Esto es así probablemente porque la política es considerada un trabajo amateur que no debería profesionalizarse (Platón, 2009). En el CSFM, la proximidad con los representados es un elemento importante de una buena representación: "Vivimos en las mismas condiciones que los colegas que representamos", insistió uno de sus miembros (Courant, 2014, 102).

El problema de sindicatos y representantes electos no estriba únicamente en su fractura con la base por no compartir las mismas experiencias vitales, sino, debido a la "ley de hierro de la oligarquía", también su ascensión a una esfera autónoma. Políticos y sindicalistas que trabajan juntos acaban convirtiéndose en colegas, creando un clima de connivencia que lleva a la desconfianza de la ciudadanía. Proudhon (2013) da testimonio de ello: "Se necesita haber vivido en esta cabina electoral que llamamos Asamblea Nacional para darse cuenta de hasta qué punto unos hombres que ignoran el estado del país son casi siempre los que lo representan". La proximidad democrática debería consistir en una forma de vida semejante a la de los representados, no la falsa "proximidad" de los políticos cuando se exhiben repartiendo apretones de manos a diestro y siniestro.

\section{D) Legitimidad}

La capacidad de algunas instituciones o individuos de poder crear obligación en un cuerpo más grande, sin el uso de presión, constituye la legitimidad. Esta consigue adhesión, haciendo que otros acepten una decisión. La legitimidad del sorteo está compuesta de tres niveles. Ya hemos visto esas tres cualidades, pero vamos a estudiarlas ahora de nuevo desde un ángulo distinto. Legitimidad es el resultado de una combinación de tres principios previos (Courant, 2014). Voy más allá del argumento dado por Dahl $(1989,342)$ y Fishkin (2003, 128) de la legitimidad democrática del mini-público: "El juicio de un mini público 'representaría' el juicio del demos".

\section{Impersonalidad: legítima por semejante}

Los representantes elegidos por sorteo son legítimos por similitud y proximidad al grupo que representan. El sorteo puede generar una muestra representativa de gran diversidad, sin el uso de cuotas si la muestra es lo suficientemente grande. Gracias a los mandatos cortos y a la rotación el sorteo está vinculado a la proximidad, al contrario que aquellos funcionarios comunistas de raíces proletarias que otrora trabajaron en fábricas a las que nunca regresaron. Existen casos de proximidad sin semejanza, como el de los jóvenes estudiantes maoístas de esmerada educación que iban a granjas y fábricas a compartir las condiciones de existencia de la clase trabajadora, sin proceder ellos mismos de familias de campesinos pobres. Lo que genera una representatividad capaz de crear adhesión es la combinación de semejanza y proximidad. Los representados puede decir: "Mi representante se parece a mí y comparte mis condiciones de vida". Ello previene también el riesgo de los líderes de 
personalidad carismática. La representatividad de la asamblea es global. La asamblea puede ser representativa únicamente como conjunto; un miembro por sí solo no puede reclamar ser un representante aislado.

\section{Horizontalidad: legítima por independiente}

¿De dónde proviene la legitimidad del sorteo? Ni desde arriba -como en el nombramiento-, ni desde abajo, como en la elección. La legitimidad del sorteo es horizontal. Mi perspectiva difiere de la de Kelsen (2004), quien comparó solamente el nombramiento y la elección, de los cuales el primero crea una dependencia de la cúpula, mientras que el segundo la crea del electorado. El sorteo produce representantes independientes que no deben su título a nadie. Esta es una buena solución para el CSFM, puesto que ni el ministro y los líderes, por un lado, y los soldados por otro, aceptarían la autoridad de una asamblea elegida por los otros (Courant, 2014). El sorteo es el único procedimiento de selección imparcial, neutral y horizontal. Los representantes seleccionados por sorteo no entran en clientelismos o electoralismos demagógicos simplemente porque no tienen que adular a un electorado para lograr salir reelegidos. No son serviles ni sumisos con aquellos que los nombraron, ni se ven obligados a obedecer reglas establecidas por expertos que diseñaron exámenes para obtener certificados.

\section{Humildad: legítima por no-superior}

Un representante elegido por un modo de selección diferente al del sorteo desarrollará una legitimidad-distinción o superioridad sobre aquellos que no fueron designados. Más aún, el representante tiene un sentimiento de superioridad personal sobre todos aquellos que ni siquiera intentaron pasar el concurso de selección; superioridad por haber ganado una elección, por ser el favorito nombrado por la cúpula o un laureado tecnócrata que ha superado a una serie de prestigiosos exámenes. Por esta razón, los elegidos no mantienen sus promesas ni escuchan las protestas populares, porque se creen mejores, superiores al Pueblo y con el derecho a gobernar. Por contraste, el sorteo es insensible al principio de distinción vinculado a la elección (Manin, 2012), así como al nombramiento y a la certificación. (Delannoi, 2010, 14) señala esta dimensión psicológica crucial:

"El sorteo no ofende a nadie", notó Montesquieu, no envanece al ganador ni llena de rencor al perdedor. Empequeñece la arrogancia y la amargura. [...] El efecto de apaciguamiento es individual, colectivo y sistémico. Casi sin excepciones. Tal vez el ganador por la suerte se vea a sí mismo como el "amado de los dioses", pero tal favor es al menos especial y nunca se posee con absoluta certidumbre. Es incomparable con el sentimiento del mérito personal.

El resto de los modos de selección ponen por delante la diferencia y la distinción y son, en consecuencia, aristocráticos. Por el contrario, el sorteo genera una legitimidad-humildad. El representante sorteado no se considera a sí mismo o a sí misma ni mejor ni peor que el resto de candidatos o que la mayoría del pueblo que ni siquiera intentó presentarse, simple- 
mente porque no hay reconocimiento, mérito, en el hecho de ser designado por la suerte. No se elige a nadie por ser diferente o superior al resto del grupo, sino porque se es parte de ese grupo en el que todos son iguales. El valor de humildad e impersonalidad confiere legitimidad al representante, que puede así afirmar: "Tengo el derecho a hablar por ti, porque nada me distingue de ti". Los representados aceptan esta afirmación representativa porque ellos mismos pueden decir del representante: "Podría haber sido yo", "puede que yo lo sea en el futuro", "él/ella es como yo", y "ella/él estuvo junto a mí compartiendo mis condiciones de vida ayer y hará lo mismo mañana", gracias a la proximidad. "El verdadero espíritu de igualdad no consiste en no querer tener un amo, sino en tener como amo a tus iguales" (Montesquieu, 1979, 245).

Por el contrario, incluso sin partidos, la elección siempre crea una distinción. Un representado puede pensar de su candidato que procede del mismo origen social y que hace un esfuerzo por aproximarse: "Mi representante se parece a mí y comparte conmigo la misma realidad cotidiana que yo... pero es diferente a mí porque ha ganado unas elecciones, algo que yo nunca lograré puesto que no poseo las cualidades necesarias para ser elegido". La misma lógica se aplica al nombramiento y a la certificación. Sin embargo, todas las personas son iguales ante el poder de la suerte, de modo que el representado puede pensar: "Si la suerte está conmigo, mañana en la asamblea puede pasar que yo ocupe el asiento de la persona que hoy me representa". La única diferencia entre las personas seleccionadas por sorteo, que deliberan en asambleas de ciudadanos o en jurados, y el resto de la población estriba en que aquellas trabajan y deliberan en estas instituciones. La causa de esto es únicamente la suerte. El único mérito de esos representantes, una vez que han sido seleccionados por sorteo, gravita en el esfuerzo que ponen en formarse y en deliberar. Pero esto está al alcance de cualquiera. Tener que realizar una selección de quiénes pueden participar en la deliberación no se debe a querer crear una elite de aristoi, sino a la necesidad de limitar el número de asambleístas para llevar adelante una deliberación en la que no pueden tomar parte, simultáneamente, millones de personas. Pero significa que los ciudadanos tal vez se pensarán participar más si el sistema los considerara a todos políticamente competentes.

El mensaje enviado por el sorteo es el siguiente: cualquiera es considerado capaz de participar directamente en deliberaciones. Es más fuerte aun acoplado a la democracia directa, como cuando una parte importante de las propuestas de los mini-públicos, como en Canadá o Irlanda, fueron sometidas al voto popular por medio de referéndum. Con su mensaje basado en la igualdad democrática y en la legitimidad-humildad, el sorteo dota de "reconocimiento" a los ciudadanos ordinarios, pudiendo llevar a un "efecto Pigmalión", a una profecía autocumplida: si a las personas se les dice que son competentes, lo más probable es que se vuelvan competentes (Rosenthal \& Jacobson, 1968). Por el contrario, la lógica de la delegación y selección de los mejores puede llevar a un "efecto Golem": a base de repetirle a las personas que no pueden participar en política sino que deben delegar su poder en otras mejores, puede ser que acaben por desmotivarse y desinteresarse completamente por la política.

El sorteo puede utilizarse para distribuir los cargos que se deseen, como en las repúblicas italianas, o para asignar a un grupo esos trabajos imprescindibles que nadie quiere hacer, como la tarea, según Rousseau (1977), de gobernar. De manera que mantener un cargo 
público no sea algo de lo que uno tenga que enorgullecerse. Además, algunos ciudadanos puede que quieran aceptar las decisiones de la asamblea sorteada simplemente porque deseen que se les acepte a ellos cuando les toque ocupar el puesto. La última parte de esta legitimidad-humildad es la "autoridad de lo ordinario", revelada en la confianza depositada en "la sabiduría popular, el sentido común del pueblo real". En ciertos casos la persona ordinaria recibe la confianza y el apoyo del grupo.

\begin{tabular}{|c|c|c|c|c|}
\hline $\begin{array}{c}\text { Marco } \\
\text { deliberativo } \\
\text { ceteris paribus }\end{array}$ & Sorteo & Elección & Certificación & Nombramiento \\
\hline $\begin{array}{l}\text { Origen de la } \\
\text { legitimidad }\end{array}$ & Neutral & $\begin{array}{l}\text { Desde abajo } \\
\text { - Elección del } \\
\text { electorado }\end{array}$ & $\begin{array}{l}\text { Quasi-cúpula } \\
\text { Examen y } \\
\text { criterios }\end{array}$ & $\begin{array}{c}\text { Desde la cúpula } \\
\text { - Elección de los } \\
\text { superiores }\end{array}$ \\
\hline $\begin{array}{l}\text { Dirección de } \\
\text { legitimidad }\end{array}$ & Horizontal & $\begin{array}{c}\text { Vertical } \\
\text { ascendente }\end{array}$ & $\begin{array}{c}\text { Semi- } \\
\text { descendente }\end{array}$ & $\begin{array}{c}\text { Vertical } \\
\text { descendente }\end{array}$ \\
\hline $\begin{array}{l}\text { Legitimidad } \\
\text { Personal }\end{array}$ & Impersonal & Muy personal & Personal & Ultra-personal \\
\hline $\begin{array}{l}\text { ¿Superioridad o } \\
\text { humildad? }\end{array}$ & Humildad & Superioridad & Superioridad & Superioridad \\
\hline Igualdad & Sí, radical & No & Relativa & No \\
\hline Imparcialidad & Sí, radical & No & Relativa & No \\
\hline Representatividad & Sí, radical & Débil & No & No \\
\hline $\begin{array}{c}\text { Tipo de } \\
\text { representación }\end{array}$ & $\begin{array}{c}\text { Espejo / } \\
\text { Descripción }\end{array}$ & Distinción & Distinción & Distinción \\
\hline $\begin{array}{c}\text { Competencia } \\
\text { examinada }\end{array}$ & No & No & Sí & No \\
\hline
\end{tabular}

\section{Conclusión}

He distinguido los modos de selección, los marcos deliberativos, es decir, lo que no depende del sorteo mismo, y los principios democráticos, los cuales son potenciales pero no tienen necesariamente que estar presentes. El marco teórico de análisis está establecido. Continuaré usándolo para encontrar en qué consiste el nuevo espíritu del sorteo y explorar su potencial para "profundizar la democracia". El sorteo no es una solución mágica y tiene unos límites, pero nos ofrece la oportunidad de pensar la democracia más allá de las elecciones, de mostrar las contradicciones entre un omnipresente pero vacío "democratismo retórico" y los principios democráticos reales. Desplaza el debate desde el par democracia directa 
versus democracia representativa, hacia la cuestión del proceso de selección de representantes, hacia democracia inclusiva versus sistema meramente electoral. La "utopía real" de la selección por sorteo lleva a reabrir la imaginación democrática y a experimentar formas más originales e inclusivas de representación, deliberación y participación.

\section{Referencias}

Aristóteles (2009): Politics, Oxford University Press, Oxford.

Bourdieu, Pierre (1981): "La représentation politique," ARSS 36-37.

Bourg, Dominique, eds., (2011): Pour une 6e République écologique, Odile Jacob, París.

Buchstein, Hubertus \& Hein, Michael (2010): "Randomizing Europe," in Gil Delannoi \& Oliver Dowlen, eds., Sortition: Theory \& Practice, Imprint Academic, Exeter.

Burdeau, George (1966 [1956]): La démocratie, Seuil, París.

Castoriadis, Cornelius (1996): La montée de l'insignifiance, Seuil, París.

Courant, Dimitri (2014): Tirage au sort et concertation dans l'Armée française: Le cas du Conseil Supérieur de la Fonction Militaire (1969-2014), Master thesis, "Etudes Politiques", EHESS, París.

Courant, Dimitri (2017): "Du klérotèrion à la cryptologie. L'acte de tirage au sort au XXIe siècle, pratiques et équipements," in Yves Sintomer \& Liliane Rabatel, eds., Tirage au sort et démocratie, La Découverte, París.

Dahl, Robert (1989): Democracy and Its Critics, Yale University Press, New Haven.

Delannoi, Gil (2010): Le retour du tirage au sort en politique, Fondapol, París.

Dewey, John (1929): The Public and its Problems, Holt, Nueva York.

Dick, Philip (1955): Solar Lottery, Ace Book, Nueva York.

Dowlen, Oliver (2008): The political potential of sortition, Imprint Acadmic, Exeter.

Dupuis-Deri, Francis (2013): Démocratie, histoire politique d'un mot, Lux, Montreal.

Fishkin, James (2003): "Consulting the Public through Deliberative Polling," Journal of Policy Analysis and Management 22, no. 1: 128-133.

Fishkin, James \& Luskin, Robert (2005): "Experimenting with a democratic ideal," Acta Politica 40: 287.

Gastil, John \& Levine, Peter, eds. (2005): The Deliberative Democracy Handbook, JosseyBass, San Francisco.

Gastil, John \& Richards, Robert (2013): "Making direct democracy deliberative through random assemblies," Politics \& Society 41, no. 2: 253-258.

Gaxie, Daniel (1993 [1978]): Le Cens caché, Seuil, París.

Hansen, Mogens H. (1991): The Athenian Democracy in the Age of Demosthenes, Blackwell, Oxford.

Kelsen, Hans (2004 [1932]): La démocratie: sa nature, sa valeur, Dalloz, París.

Kontos, Alexandros (2001 [1997]): La démocratie, un régime politique inconnu, $\mathrm{PhD}$ thesis, Paris 8, París.

Landemore, Hélène (2013): "Deliberation, cognitive diversity, and democratic inclusiveness," Synthese 190, no. 7: 1209-1231.

Lazure, Jacques (2006): Abolir les partis politiques, Libre Pensée, Montreal. 
Leib, Ethan (2004): Deliberative Democracy in America, Pennsylvania State University Press, Filadelfia.

Manin, Bernard (2012 [1995]): Principes du gouvernement représentatif, Flammarion, París.

Michels, Roberto (1971 [1911]): Les partis politiques, Flammarion, París.

Montesquieu (1979 [1748]): De l'esprit des lois, Garnier Flammarion, París.

Olson, Mancur (2011 [1965]: Logique de l'action collective, Université de Bruxelles, Bruselas. Philips, Anne (1995): The politics of presence, Clarendon Press, Oxford.

Pitkin, Hanna (1972 [1967]): The concept of representation, University of California Press, L.A.

Plato (2005): The Laws, Penguin Classics, Londres.

Plato (2009): Protagoras, Oxford University Press, Oxford.

Proudhon, Pierre-Joseph (2013 [1849]): Les Confessions d'un révolutionnaire, TOPS, París.

Rancière, Jacques (2005): La haine de la démocratie, La fabrique, París.

Reuchamps, Min \& Suiter, Jane, eds. (2016): Constitutional Deliberative Democracy in Europe, ECPR Press, Colchester.

Rosenthal, Robert \& Jacobson, Lenore (1968): "Teacher Expectation for the Disadvantaged," Scientific American 218, no. 4: 19-23.

Rousseau, Jean-Jacques (1977 [1762]): Du contrat social, Seuil, Points, París.

Saward, Michael (2010): The Representative Claim, Oxford University Press, Oxford.

Sintomer, Yves (2011): Petite histoire de l'expérimentation démocratique, La Découverte, París.

Sintomer, Yves (2013): “Les sens de la représentation politique," Raisons politiques 50, no. 2: $85-108$.

Stone, Peter (2011): The Luck of the Draw, Oxford University Press, Oxford.

Sutherland, Keith (2008 [1985]): A People's Parliament, Imprint Academic, Exeter.

Weil, Simone (1957): Note sur la suppression générale des partis politiques, Gallimard, París. 
